\title{
Primary Lymphoma of the Seminal Vesicles Presented with Acute Renal Failure: PET-CT Findings
}

\author{
Baoyi Zhu ${ }^{1}$, Yi Cai ${ }^{1}$, Ruihan Chen ${ }^{2}$, Chunwei Ye ${ }^{1}$, Yiran Tao ${ }^{1}, X$ Xingqiao Wen ${ }^{1 *}$ \\ ${ }^{1}$ Department of Urology, Third Affiliated Hospital, Sun Yat-sen University, Guangzhou, China \\ ${ }^{2}$ Department of Emergency, Third Affiliated Hospital, Sun Yat-sen University, Guangzhou, China \\ Email: *xingqiaowen@yahoo.com
}

Received May 4, 2012; revised May 28, 2012; accepted June 10, 2012

\begin{abstract}
A 63-year-old male presented with lower abdominal pain and oliguria due to a large mass in the seminal vesicles. Positron emission tomography/CT (PET/CT) examination showed multiple lesions in the seminal Vesicles, parapharyngeal space, mediastinum and spermatic cord. The result of transrectal ultrasound (TRUS)-guided biopsy indicated a diffuse, large, B-cell lymphoma of the seminal vesicles. PET-CT and TRUS guided biopsy were very helpful to make the correct diagnosis of this unique case.
\end{abstract}

Keywords: Primary Lymphoma; Seminal Vesicle; PET-CT Scan

\section{Introduction}

A 63-year-old man presented with lower abdominal pain accompanying with oliguria for 4 days. The level of serum creatinine was $727.9 \mu \mathrm{mol} / \mathrm{L}$ and the prostate specific antigen (PSA) was $2.3 \mu \mathrm{g} / \mathrm{mL}$. Magnetic resonance imaging showed a large mass lesion in the seminal vesi- cles accompanied by pelvic lymph node metastasis.

To further clarified the characteristics of the mass. Positron emission tomography/CT (PET/CT) was performed. The results showed multiple lesions in the seminal vesicles, pelvic, parapharyngeal space, mediastinum and spermatic cord (Figure 1).

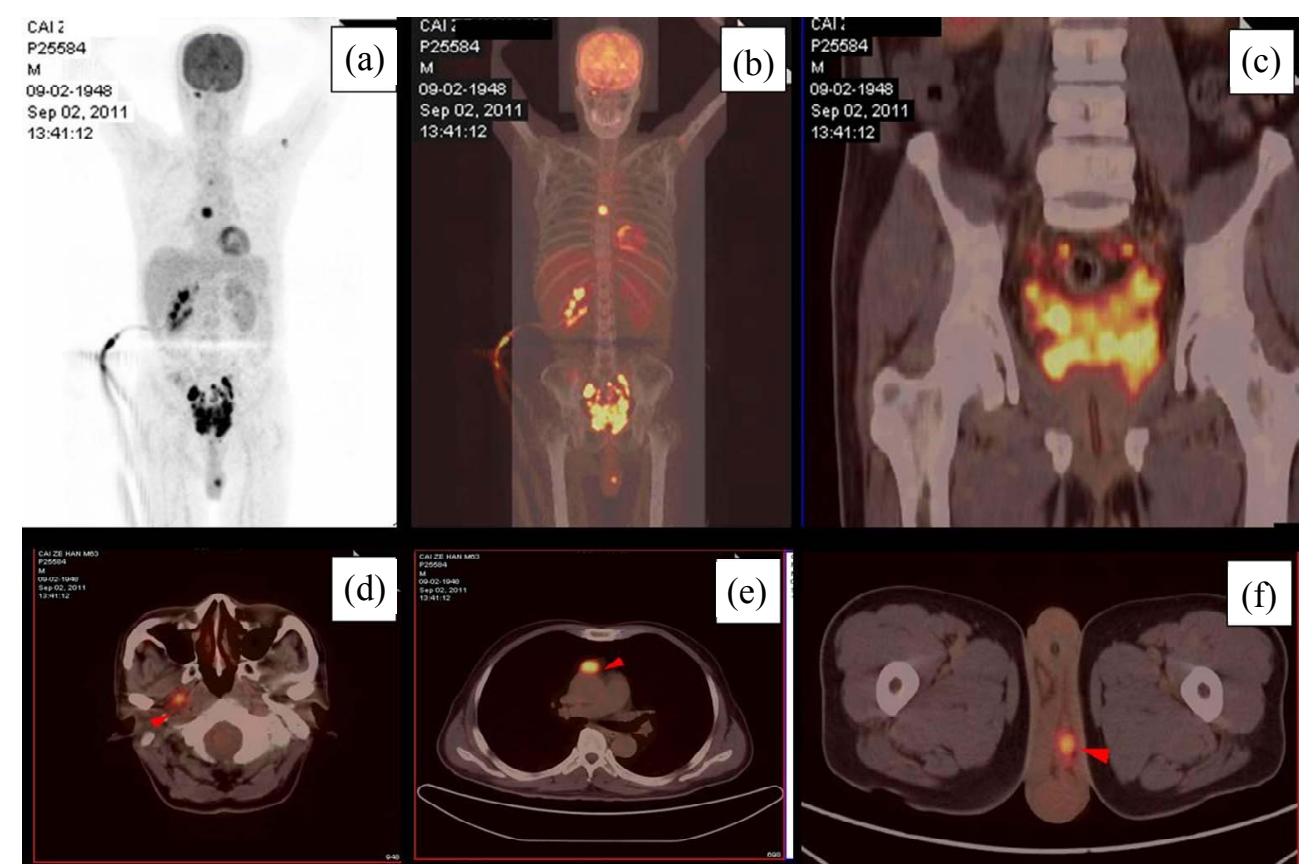

Figure 1. The PET/CT scanning of the patient showing multiple FDG-avid hotspots (a, b); especially in the seminal vesicles (c); parapharyngeal space (d); mediastinum (e) and spermatic cord (f).

\footnotetext{
"Corresponding author.
} 

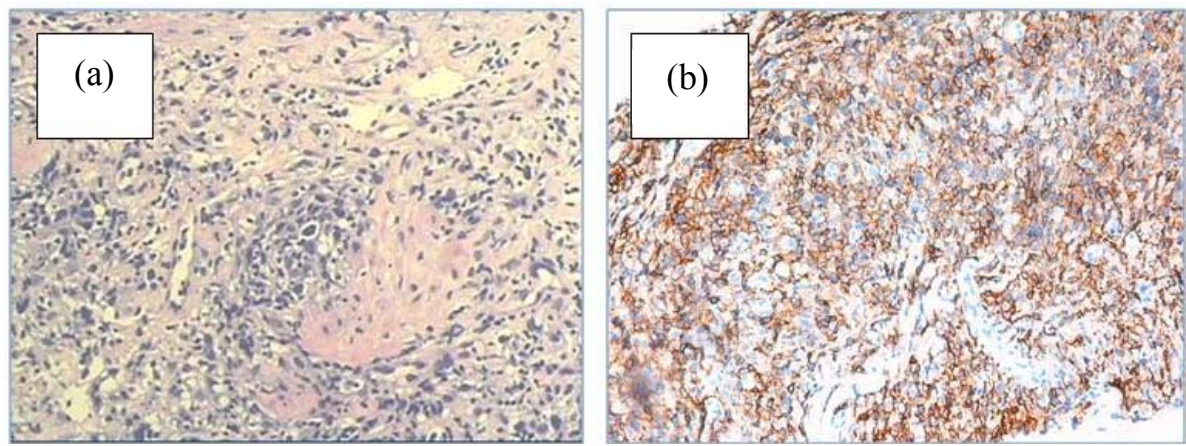

Figure 2. The tissue staining of the biopsy of seminal vesicles mass HE staining showed signs of lymphoma (a); Immunohistochemical staining showed positive of CD20 in the tissue, indicting the origin of lymphoma (b).

Based on these findings, a diagnosis of lymphoma was proposed. Transrectal ultrasound (TRUS)-guided biopsy was performed and confirmed the diagnosis of a diffuse large B-cell lymphoma (DLBCL). HE staining showed signs of lymphoma (Figure 2(a)), immunohistochemical staining showed positive of CD20 in the tissue, indicting the origin of lymphoma (Figure 2(b)). The lymphoma cells showed positive immunohistochemical staining for CD20, a specific marker of B-cell lymphoma; partial positive of CD3, CD4, CD8, CD56, CD79 $\alpha$, Vim, S100, Ki-67 and MUM-1; negative staining for CD10, CD30, CD34, P504, P63, 34BE12, PSA. He then received six cycles of R-CHOP (rituximab, cyclophosphamide, doxorubicin, vincristine and prednisone) and had achieved complete response. The serum creatinine became normal and the size of the mass decreased.

A malignant lymphoma is a malignant tumor that is derived from reticuloendothelial cells or lymphatic cells present in lymph nodes and lymphatic tissues. It mainly occurs in lymph nodes, but also occurs in other tissues in rare cases. The incidence rate of lymphomas in locations other than the primary lymph node of the pelvis, such as the retroperitoneal space, the ovary, the uterine corpus, the uterine cervix, and the vagina is known to be very low.

Primary DLBCL invasived to the seminal vesicles is rare [1]. It seldom causes severe obstruction to the lower segments of the ureters and resulted in acute renal failure. This is the first report of using PET/CT imaging to diagnosis seminal vesicles lymphoma [1]. PET-CT and TRUS guided biopsy were demonstrated to be very helpful to make the correct diagnosis of this case [2].

\section{REFERENCES}

[1] J. Zhu, et al., "Primary Diffuse Large B-Cell Lymphoma of the Seminal Vesicles: Ultrasonography and Computed Tomography Findings," Urology, Vol. 78, No. 5, 2011, pp. 1073-1074. doi:10.1016/j.urology.2011.01.032

[2] M. E. Juweid and B. D. Cheson, "Positron-Emission Tomography and Assessment of Cancer Therapy," The New England Journal of Medicine, Vol. 354, No. 5, 2006, pp. 496-507. doi:10.1056/NEJMra050276 